\title{
Distinct 1-monoacylglycerol and 2-monoacylglycerol kinase activities of diacylglycerol kinase isozymes
}

Yuriko Sato, Chiaki Murakami, Atsumi Yamaki, Satoru Mizuno, Hiromichi Sakai and Fumio Sakane*

Department of Chemistry, Graduate School of Science, Chiba University, 1-33 Yayoi-cho, Inage-ku, Chiba 263-8522, Japan

*Corresponding author. Address: Department of Chemistry, Graduate School of Science, Chiba University, 1-33 Yayoi-cho, Inage-ku, Chiba 263-8522, Japan. Tel/Fax: $+81-43-290-3695$

E-mail address: sakane@faculty.chiba-u.jp

Running title: 1-MGK and 2-MGK activities of DGKs 


\begin{abstract}
Diacylglycerolkinase (DGK) consists of ten isozymes and is involved in a wide variety ofpatho-physiological events. However, the enzymological properties of DGKs have not been fully understood. In this study, we performed a comprehensive analysis onthe 1-monoacylglycerol kinase (MGK) and 2-MGK activities of ten DGK isozymes. We revealed that type I ( $\alpha, \beta$ and $\gamma)$,type II $(\delta, \eta$ and $)$ and type III $(\varepsilon)$ DGKs have7.9-19.2\% 2-MGK activitycompared to their DGK activities, whereas their 1-MGK activities were less than 3.0\%. Both the 1-MGK and 2-MGK activities of the type IV DGKs ( $\zeta$ and $\imath$ ) were less than $1 \%$ relative totheir DGK activities. Intriguingly, type V DGK $\theta$ has approximately 6\% 1-MGK activityand less than 2\% 2-MGK activity compared to its DGK activity. Purified DGK $\theta$ exhibitedthe same results, indicating that its 1-MGK activity is intrinsic. Therefore, DGK isozymes are categorized into three types with respect to their 1-MGK and 2-MGK activities: those having (1) 2-MGK activity relatively stronger than their 1-MGK activity (types I-III), (2) only negligible 1-MGK and 2-MGK activities (type IV), and (3) 1-MGK activity stronger than its 2-MGK activity (type V). The 1-MGK activity of DGKOand the 2-MGK activity of DGK $\alpha$ were stronger than those of the acylglycerol kinase reported as 1-MGK and 2-MGK to date. The presence or absence of 1-MGK and 2-MGK activities may be essential to thepatho-physiological functions of each DGK isozyme.
\end{abstract}

\title{
Keywords
}

Diacylglycerol kinase;Monoacylglycerol kinase; Lysophosphatidic acid; Phosphatidic acid 


\section{Footnote}

\section{Abbreviations}

AGK, acylglycerol kinase; DG, diacylglycerol; DGK, diacylglycerol kinase; LPA, lysophosphatidic acid; MG, monoacylglycerol; MGK, monoacylglycerol kinase; PA, phosphatidic acid: PS, phosphatidylserine 


\section{Introduction}

Diacylglycerol kinase (DGK) phosphorylates diacylglycerol (DG) in biomembranesto produce phosphatidic acid (PA)[1-6]. To date, ten mammalian DGK isozymes $(\alpha, \beta, \gamma, \delta, \varepsilon, \zeta, \eta, \theta, 1$ and $\kappa)$ have been identified. These DGK isozymes are divided into five groups (type I: $\alpha, \beta$ and $\gamma$;type II: $\delta, \eta$ and $;$;ype III: $\varepsilon$; type IV: Cand $\mathrm{i}$; type V: $\theta$ ) according to their structural features [1-6]. DGK isozymes are known to be involved in a wide variety of patho-physiological functions.For example, DGK $\alpha$ (type I) induces clonal anergy [7, 8]. On the other hand,this isozyme also prevents melanoma apoptosis[9] and promotes hepatocellular carcinoma proliferation[10], 3D cancer cell growth [11] and angiogenesis [12]. DGK $\beta$ (type I) is an important regulator in neurite spine formation [13]. DGK $\gamma$ (type I) serves as an upstream suppressor of Rac1 and lamellipodium formation [14]. This isozyme regulates allergic reactions[15] and insulin secretion [16]. In addition to epidermal growth factor signaling [17], DGK $\delta$ (type II) is an important factor in hyperglycemia-induced peripheral insulin resistance, thereby exacerbating the severity of type-2 diabetes[18, 19]. DGKך (type II) enhances C-Raf activity and B-Raf/C-Raf heterodimerization in cancer cells[20]. It was also reported that the gene encoding DGK $\eta$ is implicated in the etiology of bipolar disorder [21]. DGKא (type II) is associated with risk of hypospadias [22]. DGKe (type III) controls seizure susceptibility and long-term potentiation through modulating arachidonoyl-inositol lipid signaling [23]. DGK $\zeta($ type IV) is an important regulator of dendritic spine maintenance[24]. This isozymeis also known to regulate endothelin-1-induced cardiomyocyte hypertrophy [25]. DGKı (type IV) regulates Ras guanyl-releasing protein 3 and inhibits Rap1 signaling [26] and presynaptic release during metabotropic glutamate receptor-dependent long-term depression [27]. DGK $\theta$ (type V) has beenimplicated in familial Parkinson disease [28] and bile acid signaling[29]. Although DGKs have been established as important biomembrane-related

modulators as described above, their enzymological properties have not been fully 
elucidated.

30 years ago, Kanohet al. purified DGKafrom porcine liver and demonstrated that the enzyme had10-20\% 2-monoacylglycerol (MG) kinase (MGK) activitycompared withits DGK activity[30]. However, its $1-\mathrm{MGK}$ activity was less than $4 \%$ relative toits DGK activity.Gantayetet al reported that DGKe also exhibited6.4\% 2-MGK activity compared withits DGK activity[31]. However, because a comprehensive analysis has not been done,the MGK activities of the other isozymes are presently unknown.

In this study, we measured the 1-MGK and 2-MGK activities of all ten DGK isozymes. We revealed that the type I DGKs $(\alpha, \beta$ and $\gamma)$, type II DGKs $(\delta, \eta$ and $\kappa)$ andtype III DGK ( $\varepsilon$ ) have 8-19\% 2-MGK activity compared to their DGK activities, whereas their 1-MGK activities were less than 3\%. Both the 2-MGK and 1-MGK activities of the type IV DGKs ( $\zeta$ and $\imath$ ) were less than $1 \%$ relative to their DGK activity. Interestingly, the type $\mathrm{V}$ DGK $\theta$ has approximately $6 \%$ 1-MGK activity and less than 2\% 2-MGK activity compared to its DGK activity.These results indicate that the ten DGK isozymes have enzymological diversity beyond our expectation. 


\section{Materials and Methods}

\subsection{Materials}

1,2-Dioleoyl-sn-glycerol,

2-arachidonoyl

glycerol, 1,2-dioleoyl-sn-glycero-3-phosphate and phosphatidylserine (PS) were purchased from Avanti Polar Lipids (Alabaster, AL). 1-Stearoyl-2-arachidonoyl-sn-glycerol, 1-oleoyl-rac-glycerol and 2-oleoylglycerol were obtained from Sigma-Aldrich (Tokyo, Japan). 1-Oleoyl lysophosphatidic acid was obtained from Cayman Chemical (Ann Arbor, MI).

\section{2. cDNA constructs}

The expression plasmids, p3xFLAG-CMV-pig DGK $\alpha[14]$, -rat DGK $\beta[14]$,

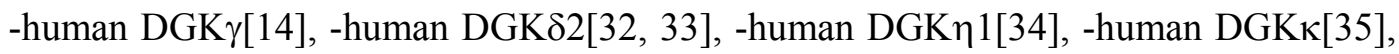
-human DGKe[36], -human DGKל1[36], -human DGKı1[36], and -human DGK $\theta[36]$ were generated as described previously. Acylglycerol kinase (AGK) cDNA was amplified by PCR from mouse muscle cDNA using the AGK forward primer: GGGAATTCGATGACTGCATTCTTTA, and the reverse primer: CCGTCGACTCACTGGGATGTGCTC. To express 6xHis-tagged protein, human DGK $\theta$ and mouse AGK cDNAs were cloned into the pSF-CMV-NH2-His-EKT3 vector (Oxford Genetics, Begbroke, UK).The amplified cDNAs were verified by sequencing carried out by Eurofins Genomics (Tokyo, Japan).

\subsection{Cell culture and transfection}

COS-7 cells were maintained in Dulbecco's modified Eagle's medium (Wako Pure Chemical Industries, Osaka, Japan) containing 10\% fetal bovine serum (Corning, Corning, NY) at $37{ }^{\circ} \mathrm{C}$ in an atmosphere containing $5 \% \mathrm{CO}_{2}$. The cells were transfected with $\mathrm{p} 3 \mathrm{xFLAG-CMV-DGK}$ isozymes by electroporation $\left(1 \times 10^{6}\right.$ cells $/ 2 \mathrm{~mm}$ gap cuvette, 110 V, 20.0 ms pulse length, one pulse) with the Gene Pulser Xcell ${ }^{\text {TM }}$ Electroporation 
System (Bio-Rad Laboratories, Tokyo, Japan) according to the manufacturer's instructions. After transfection for $48 \mathrm{~h}$, the harvested cells were suspended in $300 \mu \mathrm{l}$ ice-cold lysis buffer (50 mM HEPES (pH 7.2), $150 \mathrm{mM} \mathrm{NaCl}, 5 \mathrm{mM} \mathrm{MgCl} 2,1 \mathrm{mM}$ dithiothreitol, cOmplete ${ }^{\mathrm{TM}}$ EDTA-free protease inhibitor (Roche Diagnostics, Tokyo, Japan)) and then sonicated. The mixtures were centrifuged at $1000 \mathrm{~g}$ for $10 \mathrm{~min}$ at $4{ }^{\circ} \mathrm{C}$ to yield the cell lysates. The expression of the DGK isozymes and AGK was confirmed by Western blotting using anti-FLAG monoclonal antibody (Sigma-Aldrich, Tokyo, Japan) and anti-DGK $\theta$ monoclonal antibody (BD biosciences, San Jose, CA).

\subsection{DGK, 1-MGK and 2-MGK activity assays}

The octylglucoside mixed micellar assay of kinase activity was performed as described previously [37]. In brief, the assay mixture $(50 \mu \mathrm{l})$ contained $50 \mathrm{mM}$ MOPS (pH 7.2), $50 \mathrm{mM}$-octyl- $\beta$-D-glucoside, $1 \mathrm{mM}$ dithiothreitol, $20 \mathrm{mM} \mathrm{NaF,} 10 \mathrm{mM}$ $\mathrm{MgCl}_{2}, 27 \mathrm{~mol} \%$ PS, $5.4 \mathrm{~mol} \%$ 1,2-dioleoyl-sn-glycerol or 1-oleoyl-rac-glycerol or 2-oleoylglycerol and $1 \mathrm{mM}\left[\gamma_{-}{ }^{32} \mathrm{P}\right] \mathrm{ATP}$ (approximately $100,000 \mathrm{cpm} / \mathrm{nmol}$ ). When the activities of the $\mathrm{Ca}^{2+}$-dependent isozymes, DGK $\alpha, \beta$ and $\gamma$, were measured, $1 \mu \mathrm{M} \mathrm{CaCl}_{2}$ was added. When the activity of the arachidonoyl DG-selective isozyme, DGKe, was assessed, 1-stearoyl-2-arachidonoyl-sn-glycerol and 2-arachidonoyl glycerol were used as the substrate in place of 1,2-dioleoyl-sn-glycerol and 2-oleoylglycerol. The reaction was initiated by adding the cell lysates ( $5 \mu \mathrm{g}$ protein), and continued for $30 \mathrm{~min}$ at $30^{\circ} \mathrm{C}$. Lipids were extracted from the mixture, and PA andlysoPA (LPA) separated by thin layer chromatography were scraped and counted by a liquid scintillation spectrophotometer.

Suppl. Fig. 1shows that the expression levels of the DGK isozymes were comparable to each other.Suppl. Fig. 2 exhibitsthe DGK, 1-MGK and 2-MGK activities of the DGK isoformsthat were able to be measured above the vector control, indicating 
that they could be quantitatively analyzed. We confirmed that the assays were linear with respect to protein concentration $(0-5 \mu \mathrm{g} / 50 \mu \mathrm{l})$ and time $(0-30 \mathrm{~min})$.

\subsection{MGK activity assay in the presence of deoxycholate}

The kinase activity assay without octylglucoside was performed as described previously [38]. Lipids $(50 \mathrm{nmol})$ were dried under $\mathrm{N}_{2}$ and resuspended in $30 \mu 1$ of buffer containing $100 \mathrm{mM}$ MOPS, pH 7.2, $2 \mathrm{mM}$ EGTA, $15 \mathrm{mM} \mathrm{NaF,} 2 \mathrm{mM}$ orthovanadate, $50 \mathrm{mM} \mathrm{NaCl}, 250 \mathrm{mM}$ sucrose, $0.03 \%$ deoxycholate, and 1:5 diluted cOmplete $^{\mathrm{TM}}$ EDTA-free protease inhibitor. After a brief sonication, $10 \mu \mathrm{l}$ lysates $(5 \mu \mathrm{g})$

and $10 \mu \mathrm{l}\left[\gamma_{-}{ }^{32} \mathrm{P}\right]$ ATP $(100,000 \mathrm{cpm} / \mathrm{nmol}, 1 \mathrm{mM})$ containing $\mathrm{MgCl}_{2}(10 \mathrm{mM})$ were added, and the reactions were performed for $30 \mathrm{~min}$ at $30^{\circ} \mathrm{C}$. Lipids were extracted from the mixture, and the LPA separated by thin layer chromatography was scraped and counted by a liquid scintillation spectrophotometer.

\subsection{Purification of 6xhistidine-tagged DGK $\theta$}

$200 \mu \mathrm{l}$ Ni Sepharose 6 Fast Flow (GE Healthcare, Pittsburgh, PA) was equilibrated with $10 \mathrm{ml}$ binding buffer containing $5 \mathrm{mM}$ imidazole. The cell lysate $(2$ mg protein) was loaded onto the column, and the column was washed with $10 \mathrm{ml}$ wash buffer containing $10 \mathrm{mM}$ imidazole to remove unbound proteins. Finally, the binding protein was eluted with $800 \mu \mathrm{l}$ of elution buffer containing $50 \mathrm{mM}$ imidazole.

\subsection{Western blot analysis}

COS-7 cell lysates expressing the 3xFLAG-tagged proteins were separated using SDS-polyacrylamide gel electrophoresis. The separated proteins were transferred to a PVDF membrane (Bio-Rad Laboratories, Tokyo, Japan) and blocked with Block Ace (Dainippon Pharmaceutical, Tokyo, Japan). The membrane was incubated with an 
anti-FLAG antibody (Sigma-Aldrich) in 10\% Block Ace for $1 \mathrm{~h}$. The immunoreactive bands were then visualized using a peroxidase-conjugated anti-mouse IgG antibody (Jackson ImmunoResearch Laboratories) and the Enhanced Chemiluminescence Western Blotting Detection System (GE Healthcare). 


\section{Results}

\subsection{1-MGK and 2-MGK activities of type I DGKs ( $\alpha, \beta$ and $\gamma)$}

We first examined the 1-MGK and 2-MGK activities of a type I isozyme, DGK $\alpha$, expressed in COS-7 cells. We confirmed that the DGK activity of DGK $\alpha$ in the COS-7 cell lysates increased lineally in a time-dependent manner over $30 \mathrm{~min}$ and withthe increasing protein concentration $(0-5 \mu \mathrm{g} / 50 \mu \mathrm{l}$ of reaction mixture) [36].As shown in Fig. 1A and Table 1, DGKaexhibited $12.0 \pm 0.5 \%(n=3)$ 2-MGK activity compared to its DGK activity, whereas its 1 -MGK activity was $0.6 \pm 0.0 \%$. These results are essentially the same as those obtained with purified DGK $\alpha$ in a previous report $(10-20 \%$ of 2-MGK and $1-4 \%$ of $1-\mathrm{MGK}$ activities compared to DGK activity)[30]. Therefore, it is suggested that COS-7 cell lysates do not contain substantial DGK/1-MGK/2-MGK inhibitors or enhancers.

To confirm whether the 2-MGK activity of DGK $\alpha$ is intrinsic, we expressed 6xHis-tagged DGK $\alpha$ in COS-7 cells and purified it using Ni-affinity chromatography (Suppl. Fig. 3A). The purified DGK $\alpha$ also showed 12.0\% 2-MGK activity compared to its DGK activity, and its relative 1-MGK activity was $0.4 \%$ (Suppl. Fig. 3B). These results indicate that DGK $\alpha$ has intrinsic 2-MGK activity in addition to DGK activity.

We next compared the affinities of DGK $\alpha$ for DG and 2-MG. The activity of DGK $\alpha$ was increased in a DG or 2-MG dose-dependent manner (Suppl. Fig. 4A). A double reciprocal plot gaveapparent $K_{\mathrm{m}}$ values for DGand 2-MG of 1.4 and $11.0 \mathrm{~mol} \%$, respectively(Suppl. Fig. 4B). The apparent $K_{\mathrm{m}}$ value for 2-MG was 7.7-fold higher than that of DG.The $K_{\mathrm{m}}$ value for DG $(1.4 \mathrm{~mol} \%)$ is almost the same as that $(2.1 \mathrm{~mol} \%)$ of purified DGK $\alpha[39]$, further suggesting that the COS-7 lysates do not significantly disturb the DGK activity.

The second type I isozyme, DGK $\beta$, showed $16.8 \pm 0.3 \%$ and $1.4 \pm 0.3 \%(n=3)$ 2-MGK and 1-MGK activities, respectively, compared to its DGK activity (Fig. 1B and Table 1).Likewise, thethird type I isozyme, DGK $\gamma$,exhibited $19.2 \pm 6.3 \%$ and $0.7 \pm$ 
1.2\% 2-MGK and 1-MGK activities, respectively, compared to its DGK activity (Fig. 1C and Table 1). These results indicate that type I DGK isozymes have $12-19 \%$ 2-MGK activity relative to their DGK activities, while their 1-MGK activities are markedly lower than their 2-MGK activities $(0.6-1.4 \%)$. Of the three type I isozymes, DGK $\gamma$ has the highest relative 2-MGK activity (approximately 20\%).

\subsection{1-MGK and 2-MGK activities of type II DGKs ( $\delta$, $\eta$ and $\kappa)$}

The first type II isozyme, DGK $\delta 2$, showed $13.3 \pm 0.3 \%$ 2-MGK activity compared to its DGK activity, whereas its 1-MGK activity was $1.0 \pm 0.2 \%$ (Fig. 2A and Table 1).The same results were obtained with an alternative splicing product, DGK $\delta 1$ (data not shown). The second type II isozyme, DGK $\eta 1$,exhibited $12.6 \pm 0.3 \%$ and $3.0 \pm 0.2 \%$ 2-MGK and 1-MGK activities compared to its DGK activity (Fig. $2 \mathrm{~B}$ and Table 1), respectively.The same results were obtained with an alternative splicing product, DGK $\eta 2$ (data not shown). Similarly, the third type II isozyme, DGK and $2.0 \pm 0.9 \%$ 2-MGK and 1-MGK activitiesrelative toits DGK activity (Fig. 2C and Table 1). These results indicate that type II DGK isozymes have approximately $13 \%$ 2-MGK activity relative to their DGK activities, whereas their 1-MGK activities are markedly lower (less than 3.0\%). Therefore, the 2-MGK and 1-MGK activities of type II DGK isozymes, relative to their DGK activities are similar to those of the type I isozymes.

\subsection{1-MGK and 2-MGK activities of type III DGK $(\varepsilon)$}

The type III isozyme, DGKe, had $7.9 \pm 2.3 \%$ 2-MGK activity compared to its DGK activity (Fig. 3 and Table 1).This value is almost the same as that of a previous report by Gantayet et al. (6.4\% relative to DGK activity)[31].On the other hand, its 1-MGK activity was negligible (0.0 $\pm 0.0 \%$ DGK activity) (Fig. 3 and Table 1).We also examined DGK $\varepsilon$ activity in the absence of PS and obtained essentially the same results 
(data not shown). Therefore, similar to the type I and II isozymes, DGKe is a 2-MGK-positive and 1-MGK-negative enzyme. However, its relative 2-MGK activity was moderately lower than those of the type I and II isozymes.

\subsection{1-MGK and 2-MGK activities of type IV DGKs ( $\zeta$ and $\imath$ )}

As shown in Fig. 4A and Table 1, the 1-MGK and 2-MGK activities of the first type IV isozyme DGK $\zeta 1$ were very weak $(0.3 \pm 0.0 \%$ and $0.7 \pm 0.1 \%$ relative to DGK activity, respectively).Theactivities of the second type IV isozyme DGKı1 were also negligible $(0.0 \pm 0.0 \%$ and $0.0 \pm 0.0 \%$ relative to DGK activity, respectively) (Fig. 54B and Table 1).Therefore, in contrast to the type I, II and III DGK isozymes, type IV isozymes are 1-MGK- and 2-MGK-negative enzymes.

\subsection{1-MGK and 2-MGK activities of type VDGK $(\theta)$}

In contrast to the type I-IV DGK isozymes, DGK $\theta$ (type V) showed6.0 $\pm 0.2 \%$ 1-MGK activity compared to its DGK activity (Fig. 5 and Table 1).However, its 2-MGK activity was $1.9 \pm 0.3 \%$ relative toits DGK activity. These results indicate that, unlikethe other DGK isozymes, DGK $\theta$ is a 1-MGK-positive and 2-MGK-negative enzyme.

Compared with the ratios (12.0-19.2\%) of relative 2-MGK activities of type I and II DGK isozymes, that (6.0\%) of the 1-MGK activity of DGK $\theta$ is low. However, the comparison of the 1-MGK activity of DGK $\theta$ with the 2-MGK activity of DGKnindicates that these specific activities were comparable each other (Suppl. Fig. $5)$.

We cannot deny the possibility that the overexpression of DGK $\theta$ might induce the expression of enzyme(s) having 1-MGK activity. To confirm whetherthe 1-MGK activity of DGK $\theta$ is intrinsic, we expressed 6xHis-tagged DGK $\theta$ in COS-7 cells and purified it using Ni-affinity chromatography (Fig. 6A).The purified DGK $\theta$ also showed 4.9\% 1-MGK activity compared to its DGK activity, and its relative 2-MGK activitywas 
$0.5 \%$ (Fig. 6B). These results indicate that DGK $\theta$ possesses intrinsic 1-MGK activity in addition to DGK activity.

We next compared the affinities of DGK $\theta$ for DG and 1-MG. The activity of DGK $\theta$ was increased in a DG or 1-MG dose-dependent manner (Suppl. Fig. 6A). A double reciprocal plot provided the apparent $K_{\mathrm{m}}$ values for the DG and 1-MG of DGKӨof1.2 and $19.6 \mathrm{~mol} \%$, respectively (Suppl. Fig. 6B). The apparent $K_{\mathrm{m}}$ value for 1-MG was 16.3-fold higher than that of DG.The apparent $K_{\mathrm{m}}$ values of DGK $\theta$ for DG (1.2 mol\%) and 1-MG (19.6 mol\%) werecomparableto thoseof DGK $\alpha$ for DG and 2-MG (1.4 and $11.0 \mathrm{~mol} \%$ ), respectively (Suppl. Fig. 4).

3.6. Comparison of2-MGK and 1-MGK activities of DGK $\alpha$ and DGK $\theta$ with those of AGK

AGK was also reported to have 2-MGK and 1-MGK activities in addition to DGKactivity [38]. Thus, we compared these activities of AGK withthe 2-MGK activity of DGK $\alpha$ andthe 1-MGK activity of DGK $\theta$ using an octylglucoside-mixed micellar assay. We confirmed that the expression levels of AGK and DGK $\alpha$ were comparable to each other (Fig. 7A). As shown in Fig. 7B, DGK $\alpha$ exhibited a markedly higher 2-MGK activity (approximately 5-fold) than AGK.As shown in Fig. 8A, the expression levels of AGK and DGK $\theta$ were also comparable to each other. Moreover, DGK $\theta$ exhibited a significantly higher 1-MGK activity (approximately 11-fold) than AGK (Fig. 8B).Although the deoxycholate assay was reported to be more suitable for the measurementof the 1-MGK and 2-MGK activities of AGK than the octylglucoside-mixed micellar assay[38], we did not detect a marked enhancement of these activities of AGK, DGK $\alpha$ and DGK $\theta$ using the deoxycholate assay (data not shown). 


\section{Discussion}

30 years ago, Kanohet al. demonstrated that purified DGKahad high 2-MGK activity (10-20\% compared to DGK activity), whereas its 1-MGK activity was less than $4 \%[30]$. DGKewas also reported to have 2-MGK activity (6.4\% of DGK activity) by Gantayetet al.[31]. In the present study, DGK $\alpha$ and $\varepsilon$ exhibited approximately 12 and 8\% 2-MGK activities compared to their DGK activities (Figs. 1 and 3and Table 1). Therefore, we reproduced their results in this study. Moreover, we newly revealed that DGK $\beta, \gamma, \delta, \eta$ and $\kappa$ are also 2-MGK-positive and 1-MGK-negative enzymes (Figs. 1 and 2 and Table 1) like DGK $\alpha$ and $\varepsilon$. Among them, DGK $\gamma$ has the highest 2-MGK activity (19.2\%) relative to its DGK activity (Fig. 1). Intriguingly, we demonstrated for the first time that DGK $\theta$ has a high 1-MGK activity (approximately $5-6 \%$ of DGK activity), but not 2-MGK activity (Figs. 5 and 6 and Table 1).Notably, DGK $\zeta$ and 1 are both 1-MGK-and 2-MGK-negative (Fig. 4 and Table 1). The existence of 1-MGK- and 2-MGK-negativeisozymes emphasizes the importance of 1-MGK and 2-MGK-positive isozymes. These results indicate that the ten DGK isozymes have great enzymological diversity beyond our expectation.

We elucidated that the ten DGK isozymes have different relative 1-MGK and 2-MGK activities vs DGK activity under the same conditions. However, because the octylglucoside-mixed micellar assay was developed for DGK, it is not known whether the DGKs exhibit maximum 1-MGK and 2-MGK activities in this assay.Although the deoxycholate assay was reported to be more suitable for the measurement of the 1-MGK and 2-MGK activities of AGK than the octylglucoside-mixed micellar assay [38], we did not detect a marked enhancement of these activities of AGK and DGK using the deoxycholate assay (data not shown). If an improved MGK assay method is developed, higher intrinsic 1-MGK and 2-MGK activities of DGK isozymes may be found. 
The 1-MGK activity of DGK $\theta$ and the 2-MGK activity of DGK $\alpha$ were stronger than those of AGK (Figs. 7 and 8), which has DGK, 1-MGK and 2-MGK activities[38], under several assay conditions. Therefore, it is possible that the type I-III DGKs and type V DGK act as primary 2-MGK and 1-MGK in cells, respectively. AGK lacks a corresponding region homologous to the C-terminal half of the catalytic domain of DGK[38]. Rittineret al. [40] and we (Murakami, E. and Sakane, F. unpublished work) revealed that DGK $\eta$ lacking the C-terminal half of the catalytic domain did not show DGK activity. Therefore, this deletion may cause the low DGK, 1-MGK and 2-MGK activities of AGK.

DG content represented $0.7-4 \mathrm{~mol} \%$ of total lipids in isolated cell membranes [41].The concentrations of MG are2-10-fold higher than those of DG in macrophages[42] and 3T3-L1 adipocytes[43]. Therefore, the contribution of the 1-MGK and 2-MGK activities of DGK may be considerably great in these MG-rich cells, macrophages and adipocytes. Moreover, it is possible that the concentrations of 1-MG and 2-MG are high in a focal manner. In the 1-MG- and 2-MG-rich regions, DGK isozymes (type I-III and V) would generate substantial amounts of 1-LPA and 2-LPA.

2-Arachidonoyl-glycerol acts as endogenous cannabinoid[44, 45],so its metabolism must be strictly regulated. Because DGKeprefersDG species containing arachidonic acid at the $s n-2$ position[46, 47], this isozyme may play an important role in 2-arachidonoyl-glycerol kinase. In addition to DGK $\varepsilon[23], \mathrm{DGK} \alpha[48,49], \beta[50], \gamma[51$, $52], \delta[53]$ and $\eta[34,53,54]$ are also highly expressed in the brain.Moreover, DGK $\alpha, \beta, \gamma$, $\delta$ and $\eta$ have broadselectivities to fatty acids at the sn-2 position of DG[3]. For example, DGK $\gamma$ indeed showedcomparable phosphorylation activitiesagainst 2-oleoyl(18:1)-glycerol and 2-arachidonoyl-glycerol (Suppl. Fig. 7).Therefore, these type I and II isozymes may also act as 2-arachidonoyl-glycerol kinase in the brain.

Our preliminary experiments showed that DGK $\delta$-specific siRNAattenuateda high glucose-dependent increase of LPA (Sakai, H. and Sakane, F., unpublished work). 
However, a phospholipase A inhibitor (methyl arachidonylfluorophosphonate) [55, 56]did not inhibitthe high glucose-dependent increase of LPA (Sato, Y., Mizuno, S.and Sakane, F. unpublished work). These results suggest that DGK $\delta$ produces LPA through the direct phosphorylation of MG, but not the PA hydrolysis by phospholipase A. Thus, although further works are needed to confirm, it is possible that DGK $\delta$ serves as MGK in cells.

The ten DGK isozymes are divided into five groups (type I: $\alpha, \beta$ and $\gamma$; type II:

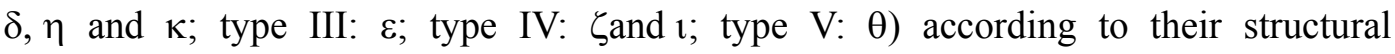
features[1-6].Arachidonic acid-containing DG-selectivity can also be used to categorize the isozymes into two groups: an arachidonoyl DG-selective isozyme $(\varepsilon)$ versus non-selective isozymes $(\alpha, \beta, \gamma, \delta, \eta, \kappa, \zeta$, tand $\theta)$ [1-6]. DGK isozymes can also be grouped into high DG affinity type $(\eta)$ versus low DG affinity type $(\alpha, \varepsilon$ and $\zeta)$ (the affinities of the other isozymes for DG are presently unknown)[57]. In addition to these categorizations, the results of this study provide a new classification based on the different activities for 1-MG and 2-MG: DGK isozyme(s) having (1) 2-MGK activity relatively stronger than their 1-MGK activity (type I-III DGKs), (2) only negligible 1-MGK and 2-MGK activities (type IV DGK), and (3) 1-MGK activity stronger than its 2-MGK (type V DGK). These differencesin regard to the relative 1-MGK and 2-MGKactivities are probably essential for the DGK isozymes in serving their individual functions.

LPA is known to regulate the peroxisome proliferator-activated receptor $\gamma$ andthe reorganization of actin cytoskeleton[58]. However, the physiological functions of LPA in cells are still poorly understood. The simultaneous production of PA with/without 1-LPA or 2-LPA may be important to maximize a variety of physiological functions of DGKs[1-6]. This study provides a new aspect of DGK and will enhance the elucidation of the physiological functions of LPA in cells. 


\section{Acknowledgments}

This work was supported by MEXT/JSPS KAKENHI Grant Numbers 22370047 (Grant-in-Aid for Scientific Research (B)), 23116505 (Grant-in-Aid for Scientific Research on Innovative Areas), 25116704 (Grant-in-Aid for Scientific Research on Innovative Areas), 26291017 (Grant-in-Aid for Scientific Research (B)) and 15K14470 (Grant-in-Aid for Challenging Exploratory Research), the Japan Science and Technology Agency (AS221Z00794F, AS231Z00139G, AS251Z01788Q and AS2621643Q), the Naito Foundation, the Hamaguchi Foundation for the Advancement of Biochemistry, the Daiichi-Sankyo Foundation of Life Science, the Terumo Life Science Foundation, the Futaba Electronic Memorial Foundation, the Daiwa Securities Health Foundation, the Ono Medical Research Foundation, the Japan Foundation for Applied Enzymology, the Food Science Institute Foundation, the Skylark Food Science Institute, the Asahi Group Foundation and the Venture Business Laboratory of Chiba University (FS).

\section{Author contributions}

FS conceived and coordinated the study and wrote the paper. YS, CM, AY, SM and HS designed, performed and analyzed the experiments. 


\section{References}

[1] K. Goto, Y. Hozumi, H. Kondo, Diacylglycerol, phosphatidic acid, and the converting enzyme, diacylglycerol kinase, in the nucleus, Biochim. Biophys. Acta, 1761 (2006) 535-541.

[2] I. Merida, A. Avila-Flores, E. Merino, Diacylglycerol kinases: at the hub of cell signalling, Biochem. J., 409 (2008) 1-18.

[3] F. Sakane, S. Imai, M. Kai, S. Yasuda, H. Kanoh, Diacylglycerol kinases: why so many of them?, Biochim. Biophys. Acta, 1771 (2007) 793-806.

[4] F. Sakane, S. Imai, M. Kai, S. Yasuda, H. Kanoh, Diacylglycerol kinases as emerging potential drug targets for a variety of diseases, Curr. Drug Targets, 9 (2008) 626-640.

[5] M.K. Topham, Signaling roles of diacylglycerol kinases, J. Cell. Biochem., 97 (2006) 474-484.

[6] W.J. van Blitterswijk, B. Houssa, Properties and functions of diacylglycerol kinases, Cell. Signal., 12 (2000) 595-605.

[7] B.A. Olenchock, R. Guo, J.H. Carpenter, M. Jordan, M.K. Topham, G.A. Koretzky, X.P. Zhong, Disruption of diacylglycerol metabolism impairs the induction of $\mathrm{T}$ cell anergy, Nat. Immunol., 7 (2006) 1174-1181.

[8] Y. Zha, R. Marks, A.W. Ho, A.C. Peterson, S. Janardhan, I. Brown, K. Praveen, S. Stang, J.C. Stone, T.F. Gajewski, T cell anergy is reversed by active Ras and is regulated by diacylglycerol kinase- $\alpha$, Nat. Immunol., 7 (2006) 1166-1173.

[9] K. Yanagisawa, S. Yasuda, M. Kai, S. Imai, K. Yamada, T. Yamashita, K. Jimbow, H. Kanoh, F. Sakane, Diacylglycerol kinase $\alpha$ suppresses tumor necrosis factor- $\alpha$-induced apoptosis of human melanoma cells through NF- $\kappa \mathrm{B}$ activation, Biochim. Biophys. Acta, 1771 (2007) 462-474.

[10] K. Takeishi, A. Taketomi, K. Shirabe, T. Toshima, T. Motomura, T. Ikegami, T. Yoshizumi, F. Sakane, Y. Maehara, Diacylglycerol kinase alpha enhances hepatocellular carcinoma progression by activation of Ras-Raf-MEK-ERK pathway, Journal of hepatology, 57 (2012) 77-83.

[11] P. Torres-Ayuso, M. Daza-Martin, J. Martin-Perez, A. Avila-Flores, I. Merida, Diacylglycerol kinase $\alpha$ promotes 3D cancer cell growth and limits drug sensitivity through functional interaction with Src, Oncotarget, 5 (2014) 9710-9726. 
[12] G. Baldanzi, S. Mitola, S. Cutrupi, N. Filigheddu, W.J. van Blitterswijk, F. Sinigaglia, F. Bussolino, A. Graziani, Activation of diacylglycerol kinase $\alpha$ is required for VEGF-induced angiogenic signaling in vitro, Oncogene, 23 (2004) 4828-4838.

[13] Y. Shirai, T. Kouzuki, K. Kakefuda, S. Moriguchi, A. Oyagi, K. Horie, S.Y. Morita, M. Shimazawa, K. Fukunaga, J. Takeda, N. Saito, H. Hara, Essential role of neuron-enriched diacylglycerol kinase (DGK), DGK $\beta$ in neurite spine formation, contributing to cognitive function, PLoS One, 5 (2010) e11602.

[14] S. Tsushima, M. Kai, K. Yamada, S. Imai, K. Houkin, H. Kanoh, F. Sakane, Diacylglycerol kinase $\gamma$ serves as an upstream suppressor of Rac1 and lamellipodium formation, J. Biol. Chem., 279 (2004) 28603-28613.

[15] M. Sakuma, Y. Shirai, T. Ueyama, N. Saito, Diacylglycerol kinase gamma regulates antigen-induced mast cell degranulation by mediating $\mathrm{Ca}(2+)$ influxes, Biochem Biophys Res Commun, 445 (2014) 340-345.

[16] Y. Kurohane Kaneko, Y. Kobayashi, K. Motoki, K. Nakata, S. Miyagawa, M. Yamamoto, D. Hayashi, Y. Shirai, F. Sakane, T. Ishikawa, Depression of Type I Diacylglycerol Kinases in Pancreatic $\beta$-Cells From Male Mice Results in Impaired Insulin Secretion, Endocrinology, 154 (2013) 4089-4098.

[17] T. Crotty, J. Cai, F. Sakane, A. Taketomi, S.M. Prescott, M.K. Topham, Diacylglycerol kinase $\delta$ regulates protein kinase $\mathrm{C}$ and epidermal growth factor receptor signaling, Proc. Natl. Acad. Sci. U S A, 103 (2006) 15485-15490.

[18] A.V. Chibalin, Y. Leng, E. Vieira, A. Krook, M. Bjornholm, Y.C. Long, O. Kotova, Z. Zhong, F. Sakane, T. Steiler, C. Nylen, J. Wang, M. Laakso, M.K. Topham, M. Gilbert, H. Wallberg-Henriksson, J.R. Zierath, Downregulation of diacylglycerol kinase delta contributes to hyperglycemia-induced insulin resistance, Cell, 132 (2008) 375-386.

[19] C. Miele, F. Paturzo, R. Teperino, F. Sakane, F. Fiory, F. Oriente, P. Ungaro, R. Valentino, F. Beguinot, P. Formisano, Glucose regulates diacylglycerol intracellular levels and protein kinase $\mathrm{C}$ activity by modulating diacylglycerol-kinase subcellular localization, J. Biol. Chem., 282 (2007) 31835-31843.

[20] S. Yasuda, M. Kai, S. Imai, K. Takeishi, A. Taketomi, M. Toyota, H. Kanoh, F. Sakane, Diacylglycerol kinase $\eta$ augments C-Raf activity and B-Raf/C-Raf 
heterodimerization, J. Biol. Chem., 284 (2009) 29559-29570.

[21] A.E. Baum, N. Akula, M. Cabanero, I. Cardona, W. Corona, B. Klemens, T.G. Schulze, S. Cichon, M. Rietschel, M.M. Nothen, A. Georgi, J. Schumacher, M. Schwarz, R. Abou Jamra, S. Hofels, P. Propping, J. Satagopan, S.D. Detera-Wadleigh, J. Hardy, F.J. McMahon, A genome-wide association study implicates diacylglycerol kinase eta (DGKH) and several other genes in the etiology of bipolar disorder, Mol. Psychiatry, 13 (2008) 197-207.

[22] L.F. van der Zanden, I.A. van Rooij, W.F. Feitz, J. Knight, A.R. Donders, K.Y. Renkema, E.M. Bongers, S.H. Vermeulen, L.A. Kiemeney, J.A. Veltman, A. Arias-Vasquez, X. Zhang, E. Markljung, L. Qiao, L.S. Baskin, A. Nordenskjold, N. Roeleveld, B. Franke, N.V. Knoers, Common variants in DGKK are strongly associated with risk of hypospadias, Nat. Genet., 43 (2011) 48-50.

[23] E.B. Rodriguez De Turco, W. Tang, M.K. Topham, F. Sakane, V.L. Marcheselli, C. Chen, A. Taketomi, S.M. Prescott, N.G. Bazan, Diacylglycerol kinase $\varepsilon$ regulates seizure susceptibility and long-term potentiation through arachidonoyl- inositol lipid signaling, Proc. Natl. Acad. Sci. USA, 98 (2001) 4740-4745.

[24] K. Kim, J. Yang, X.P. Zhong, M.H. Kim, Y.S. Kim, H.W. Lee, S. Han, J. Choi, K. Han, J. Seo, S.M. Prescott, M.K. Topham, Y.C. Bae, G. Koretzky, S.Y. Choi, E. Kim, Synaptic removal of diacylglycerol by DGK $\zeta$ and PSD-95 regulates dendritic spine maintenance, EMBO J., 28 (2009) 1170-1179.

[25] H. Takahashi, Y. Takeishi, T. Seidler, T. Arimoto, H. Akiyama, Y. Hozumi, Y. Koyama, T. Shishido, Y. Tsunoda, T. Niizeki, N. Nozaki, J. Abe, G. Hasenfuss, K. Goto, I. Kubota, Adenovirus-mediated overexpression of diacylglycerol kinase-zeta inhibits endothelin-1-induced cardiomyocyte hypertrophy, Circulation, 111 (2005) $1510-1516$.

[26] D.S. Regier, J. Higbee, K.M. Lund, F. Sakane, S.M. Prescott, M.K. Topham, Diacylglycerol kinase 1 regulates Ras guanyl-releasing protein 3 and inhibits Rap1 signaling, Proc. Natl. Acad. Sci. U S A, 102 (2005) 7595-7600.

[27] J. Yang, J. Seo, R. Nair, S. Han, S. Jang, K. Kim, K. Han, S.K. Paik, J. Choi, S. Lee, Y.C. Bae, M.K. Topham, S.M. Prescott, J.S. Rhee, S.Y. Choi, E. Kim, DGKı regulates presynaptic release during mGluR-dependent LTD, EMBO J., 30 (2011) 165-180. 
[28] N. Pankratz, J.B. Wilk, J.C. Latourelle, A.L. DeStefano, C. Halter, E.W. Pugh, K.F. Doheny, J.F. Gusella, W.C. Nichols, T. Foroud, R.H. Myers, Genomewide association study for susceptibility genes contributing to familial Parkinson disease, Hum. Genet., 124 (2009) 593-605.

[29] K. Cai, M.B. Sewer, Diacylglycerol kinase $\theta$ couples farnesoid X receptor-dependent bile acid signalling to Akt activation and glucose homoeostasis in hepatocytes, Biochem. J., 454 (2013) 267-274.

[30] H. Kanoh, T. Iwata, T. Ono, T. Suzuki, Immunological characterization of sn-1,2-diacylglycerol and sn-2-monoacylglycerol kinase from pig brain, J. Biol. Chem., 261 (1986) 5597-5602.

[31] A. Gantayet, J. Jegatheswaran, G. Jayakumaran, M.K. Topham, R.M. Epand, Endocannabinoids and diacylglycerol kinase activity, Biochim Biophys Acta, 1808 (2011) 1050-1053.

[32] S. Imai, F. Sakane, H. Kanoh, Phorbol ester-regulated oligomerization of diacylglycerol kinase $\delta$ linked to its phosphorylation and translocation, J. Biol. Chem., 277 (2002) 35323-35332.

[33] F. Sakane, S. Imai, K. Yamada, T. Murakami, S. Tsushima, H. Kanoh, Alternative splicing of the human diacylglycerol kinase $\delta$ gene generates two isoforms differing in their expression patterns and in regulatory functions, J. Biol. Chem., 277 (2002) 43519-43526.

[34] T. Murakami, F. Sakane, S. Imai, K. Houkin, H. Kanoh, Identification and characterization of two splice variants of human diacylglycerol kinase $\eta$, J. Biol. Chem., 278 (2003) 34364-34372.

[35] S. Imai, M. Kai, S. Yasuda, H. Kanoh, F. Sakane, Identification and

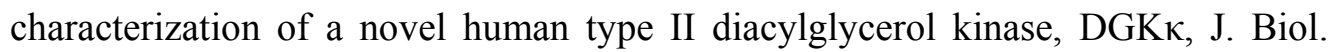
Chem., 280 (2005) 39870-39881.

[36] M. Sato, K. Liu, S. Sasaki, N. Kunii, H. Sakai, H. Mizuno, H. Saga, F. Sakane, Evaluations of the selectivities of the diacylglycerol kinase inhibitors R59022 and R59949 among diacylglycerol kinase isozymes using a new non-radioactive assay method, Pharmacology, 92 (2013) 99-107.

[37] F. Sakane, S. Imai, M. Kai, I. Wada, H. Kanoh, Molecular cloning of a novel diacylglycerol kinase isozyme with a pleckstrin homology domain and a C-terminal 
tail similar to those of the EPH family of protein tyrosine kinase, J. Biol. Chem., 271 (1996) 8394-8401.

[38] M. Bektas, S.G. Payne, H. Liu, S. Goparaju, S. Milstien, S. Spiegel, A novel acylglycerol kinase that produces lysophosphatidic acid modulates cross talk with EGFR in prostate cancer cells, J Cell Biol, 169 (2005) 801-811.

[39] Y. Jiang, F. Sakane, H. Kanoh, J.P. Walsh, Selectivity of the diacylglycerol kinase inhibitor 3-2-(4-[bis-(4-fluorophenyl)methylene]-1-piperidinyl)ethyl-2, 3-dihydro-2-thioxo-4(1H)quinazolinone (R59949) among diacylglycerol kinase subtypes, Biochem. Pharmacol., 59 (2000) 763-772.

[40] J.E. Rittiner, V.E. Brings, M.J. Zylka, Overexpression of diacylglycerol kinase eta enhances Galphaq-coupled G protein-coupled receptor signaling, Mol Pharmacol, 85 (2014) 800-810.

[41] Y. Ueda, R. Ishitsuka, F. Hullin-Matsuda, T. Kobayashi, Regulation of the transbilayer movement of diacylglycerol in the plasma membrane, Biochimie, 107 Pt A (2014) 43-50.

[42] T.J. Leiker, R.M. Barkley, R.C. Murphy, Analysis of Diacylglycerol Molecular Species in Cellular Lipid Extracts by Normal-Phase LC-Electrospray Mass Spectrometry, International journal of mass spectrometry, 305 (2011) 103-109.

[43] S. Lobo, B.M. Wiczer, D.A. Bernlohr, Functional analysis of long-chain acyl-CoA synthetase 1 in 3T3-L1 adipocytes, J Biol Chem, 284 (2009) 18347-18356.

[44] V. Di Marzo, L. De Petrocellis, T. Sugiura, K. Waku, Potential biosynthetic connections between the two cannabimimetic eicosanoids, anandamide and 2-arachidonoyl-glycerol, in mouse neuroblastoma cells, Biochem Biophys Res Commun, 227 (1996) 281-288.

[45] T. Sugiura, K. Waku, 2-Arachidonoylglycerol and the cannabinoid receptors, Chem Phys Lipids, 108 (2000) 89-106.

[46] M.L. MacDonald, K.F. Mack, B.W. Williams, W.C. King, J.A. Glomset, A membrane-bound diacylglycerol kinase that selectively phosphorylates arachidonoyl-diacylglycerol. Distinction from cytosolic diacylglycerol kinase and comparison with the membrane-bound enzyme from Escherichia coli., J. Biol. Chem., 263 (1988) 1584-1592.

[47] W. Tang, M. Bunting, G.A. Zimmerman, T.M. McIntyre, S.M. Prescott, Molecular 
cloning of a novel human diacylglycerol kinase highly selective for arachidonate-containing substrates, J. Biol. Chem., 271 (1996) 10237-10241.

[48] K. Goto, M. Watanabe, H. Kondo, H. Yuasa, F. Sakane, H. Kanoh, Gene cloning, sequence, expression and in situ localization of $80 \mathrm{kDa}$ diacylglycerol kinase specific to oligodendrocyte of rat brain, Mol. Brain Res., 16 (1992) 75-87.

[49] F. Sakane, K. Yamada, H. Kanoh, C. Yokoyama, T. Tanabe, Porcine diacylglycerol kinase sequence has zinc finger and E-F hand motifs, Nature, 344 (1990) 345-348.

[50] K. Goto, H. Kondo, Molecular cloning and expression of a 90-kDa diacylglycerol kinase that predominantly localizes in neurons, Proc. Natl. Acad. Sci. USA, 90 (1993) 7598-7602.

[51] K. Goto, M. Funayama, H. Kondo, Cloning and expression of a cytoskeleton-associated diacylglycerol kinase that is dominantly expressed in cerebellum, Proc. Natl. Acad. Sci. USA, 91 (1994) 13042-13046.

[52] M. Kai, F. Sakane, S. Imai, I. Wada, H. Kanoh, Molecular cloning of a diacylglycerol kinase isozyme predominantly expressed in human retina with a truncated and inactive enzyme expression in most other human cells, J. Biol. Chem., 269 (1994) 18492-18498.

[53] T. Usuki, H. Sakai, T. Shionoya, N. Sato, F. Sakane, Expression and localization of type II diacylglycerol kinase isozymes delta and eta in the developing mouse brain, The journal of histochemistry and cytochemistry : official journal of the Histochemistry Society, 63 (2015) 57-68.

[54] T.M. Klauck, X. Xu, B. Mousseau, S. Jaken, Cloning and characterization of a glucocorticoid-induced diacylglycerol kinase, J. Biol. Chem., 271 (1996) 19781-19788.

[55] P. Rastogi, C.S. Beckett, J. McHowat, Prostaglandin production in human coronary artery endothelial cells is modulated differentially by selective phospholipase A(2) inhibitors, Prostaglandins, leukotrienes, and essential fatty acids, 76 (2007) 205-212.

[56] A. Yamashita, T. Kumazawa, H. Koga, N. Suzuki, S. Oka, T. Sugiura, Generation of lysophosphatidylinositol by DDHD domain containing 1 (DDHD1): Possible involvement of phospholipase D/phosphatidic acid in the activation of DDHD1, Biochim Biophys Acta, 1801 (2010) 711-720. 
[57] S. Komenoi, F. Takemura, H. Sakai, F. Sakane, Diacylglycerol kinase eta1 is a high affinity isozyme for diacylglycerol, FEBS Lett., 589 (2015) 1272-1277.

[58] G. Tigyi, Aiming drug discovery at lysophosphatidic acid targets, British journal of pharmacology, 161 (2010) 241-270. 


\section{Figure Legends}

Fig. 1. MGK activities of type I DGK isozymes. 1,000xg supernatants (5 $\mu \mathrm{g})$ of extracts from COS-7 cells expressing each DGK isozyme ( $\alpha(\mathrm{A}), \beta(\mathrm{B})$ and $\gamma(\mathrm{C}))$ were incubated with DG, 1-MG and 2-MG for $30 \mathrm{~min}$ in the presence of $1 \mu \mathrm{M} \mathrm{CaCl}_{2}$. The results are presented as percentages of the value of the control (DGK activity) and represent the means \pm SDs of the values obtained in three separate experiments. The statistical significance was determined using Student's t test $(* * * p<0.005)$.

Fig. 2. MGK activities of type II DGK isozymes.1,000xg supernatants $(5 \mu \mathrm{g})$ of extracts from COS-7 cells expressing each DGK isozyme ( $\delta 2(\mathrm{~A}), \eta 1(\mathrm{~B})$ and $\kappa(\mathrm{C}))$ were incubated with $\mathrm{DG}, 1-\mathrm{MG}$ and 2-MG for $30 \mathrm{~min}$. The results are presented as percentages of the value of the control (DGK activity) and represent the means \pm SDs of the values obtained in three separate experiments. The statistical significance was determined using Student's t test $(* * * p<0.005)$.

Fig. 3. MGK activity of type III DGK isozyme. 1,000xg supernatants $(5 \mu \mathrm{g})$ of extracts from COS-7 cells expressing type III DGK isozyme $(\varepsilon)$ were incubated with DG, 1-MG and 2-MG for $30 \mathrm{~min}$. Because DGKe is the 2-arachidonoyl DG-selective isozyme, 1-stearoly-2-arachidonoyl-sn-glycerol and 2-arachdonyl glycerol were used as substrates in place of 1,2-dioleoyl-sn-glycerol and 2-oleoylglycerol. The resultsare presented as percentages of the value of the control (DGK activity) and represent the means \pm SDs of the values obtained in three separate experiments. The statistical significance was determined using Student's t test $(* * * p<0.005)$.

Fig. 4. MGK activities of type IV DGK isozymes. (A, B) 1,000xg supernatants (5 $\mu \mathrm{g})$ of extracts from COS-7 cells expressing type IV DGK isozymes ( $\zeta 1$ (A) and $\imath 1$ (B)) 
were incubated with $\mathrm{DG}, 1-\mathrm{MG}$ and $2-\mathrm{MG}$ for $30 \mathrm{~min}$. The resultsare presented as percentages of the value of the control (DGK activity) and represent the means \pm SDs of the values obtained in three separate experiments. The statistical significance was determined using Student's t test $(* * * p<0.005)$.

Fig. 5. MGK activity of type V DGK isozyme. 1,000xg supernatants (5 $\mu \mathrm{g})$ of extracts from COS-7 cells expressing type V DGK isozyme $(\theta)$ was incubated with DG, 1-MG and $2-\mathrm{MG}$ for $30 \mathrm{~min}$. The resultsare presented as percentages of the value of the control (DGK activity), and represent the means \pm SDs of the values obtained in three separate experiments. The statistical significance was determined using Student's t test $(* * * p<0.005)$.

Fig. 6. 1-MGK activity of purified DGK $\theta$. (A) 6xHis-tagged DGK $\theta$ was purified by Ni Sepharose affinity chromatography. The 6xHis-tagged DGK $\theta$ in each fraction was detected by Western blotting using an anti-DGK $\theta$ monoclonal antibody. (B) Kinase activities of purified DGK $\theta$ for DG, 1-MG and 2-MG were examined. The results are presented as percentages of the value of the control (DGK activity) and represent the means \pm SDs of the values obtained in three separate experiments. The statistical significance was determined using Student's t test $(* * * p<0.005)$.

Fig. 7. 2-MGK activities of AGK and DGKa.(A) The expression of the 3xFLAG-tagged AGK and DGK $\alpha$. The 3xFLAG-tagged AGK and DGK $\alpha$ expressed in COS-7 cells were detected by Western blotting using an anti-FLAG monoclonal antibody. (B) 1,000xg supernatants $(5 \mu \mathrm{g})$ of extracts from COS-7 cells expressing 3xFLAG-AGK and 3xFLAG-DGK $\alpha$ were incubated with 2-MG for $30 \mathrm{~min}$. The results are presented as relativeto the control (2-MG kinase activity of 3xFLAG-AGK). The 2-MGK activity of 3xFLAG-DGK $\alpha$ was normalized for the expression levels of 
3xFLAG-AGK.Representative results of three independent experimentsare shown.

Fig. 8. 1-MGK activities of AGK and DGK $\theta$.(A) The expression of the $3 x F L A G$-tagged AGK and DGK $\theta$. The 3xFLAG-tagged AGK and DGK $\theta$ expressed in COS-7 cells were detected by Western blotting using an anti-FLAG monoclonal antibody. (B) 1,000xg supernatants $(5 \mu \mathrm{g})$ of extracts from COS-7 cells expressing 3xFLAG-AGK and $3 \mathrm{xFLAG-DGK} \theta$ were incubated with $1-\mathrm{MG}$ for $30 \mathrm{~min}$. The results are presented as relativeto the control (1-MGK activity of 3xFLAG-AGK). The 1-MGK activity of $3 x$ FLAG-DGK $\theta$ was normalized for the expression levels of 3xFLAG-AGK.Representative results of three independent experimentsare shown. 
Table 1

1-MGK and 2-MGK activities of ten DGK isozymes

\begin{tabular}{|c|c|c|c|c|}
\hline \multirow{2}{*}{ Type } & \multirow{2}{*}{ Isozyme } & \multicolumn{3}{|c|}{ Relative activity (\%) } \\
\cline { 2 - 5 } & & DG & 1-MG & 2-MG \\
\hline \multirow{2}{*}{ I } & $\alpha$ & 100 & $0.6 \pm 0.0$ & $12.0 \pm 0.5$ \\
\cline { 2 - 5 } & $\beta$ & 100 & $1.4 \pm 0.3$ & $16.8 \pm 0.3$ \\
\cline { 2 - 5 } & $\gamma$ & 100 & $0.7 \pm 1.2$ & $19.2 \pm 6.3$ \\
\hline \multirow{2}{*}{ II } & $\delta 2$ & 100 & $1.0 \pm 0.2$ & $13.3 \pm 0.3$ \\
\cline { 2 - 5 } & $\eta 1$ & 100 & $3.0 \pm 0.2$ & $12.6 \pm 0.3$ \\
\cline { 2 - 5 } & $\kappa$ & 100 & $2.0 \pm 0.9$ & $12.4 \pm 2.4$ \\
\hline \multirow{2}{*}{ III } & $\zeta 1$ & 100 & $0.0 \pm 0.0$ & $7.9 \pm 2.3$ \\
\hline \multirow{2}{*}{ IV } & 11 & 100 & $0.3 \pm 0.0$ & $0.7 \pm 0.1$ \\
\hline & $\theta$ & 100 & $0.0 \pm 0.0$ & $0.0 \pm 0.1$ \\
\hline
\end{tabular}

$1,000 \mathrm{xg}$ supernatants $(5 \mu \mathrm{g})$ of extracts from COS-7 cells expressing each DGK isozyme $(\alpha-\theta)$ were incubated with $\mathrm{DG}, 1-\mathrm{MG}$ and $2-\mathrm{MG}$ for $30 \mathrm{~min}$, and the kinase activities of the DGK isozyme for DG, 1-MG and 2-MG were examined (Figs. 1-5). The data are shown as the percentages of the value of the control (DGK activity), and the means \pm SDs of the values obtained in three separate experiments. 


\begin{tabular}{|c|c|c|c|c|}
\hline \multicolumn{2}{|c|}{ DG } & \multirow{2}{*}{$\begin{array}{c}\text { Type I, II, III } \\
\text { DGK } \\
\stackrel{(\alpha, \beta, \gamma, \delta, \eta, \kappa, \varepsilon)}{\longrightarrow}\end{array}$} & \multicolumn{2}{|c|}{ PA } \\
\hline 1-MG & 2-MG & & 1-LPA & 2-LPA \\
\hline
\end{tabular}
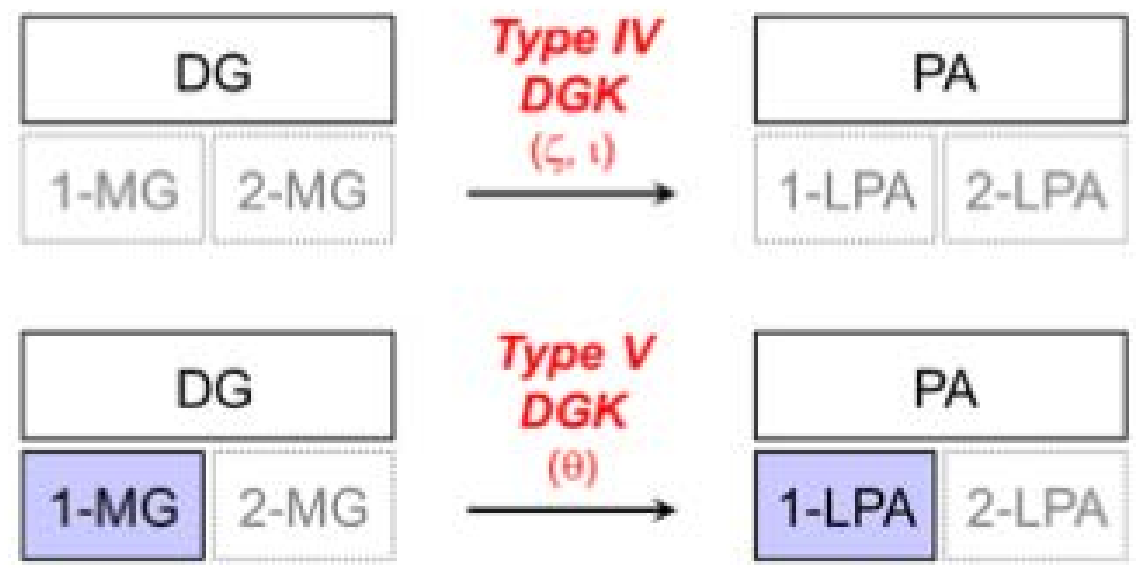
Fig. 1

A

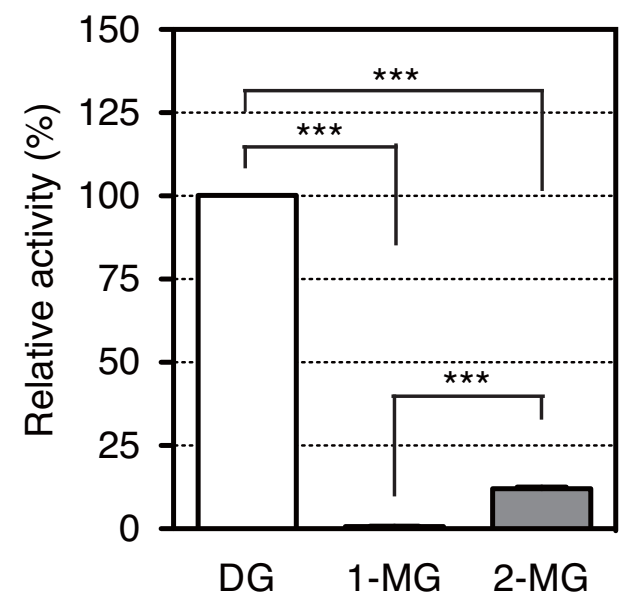

B

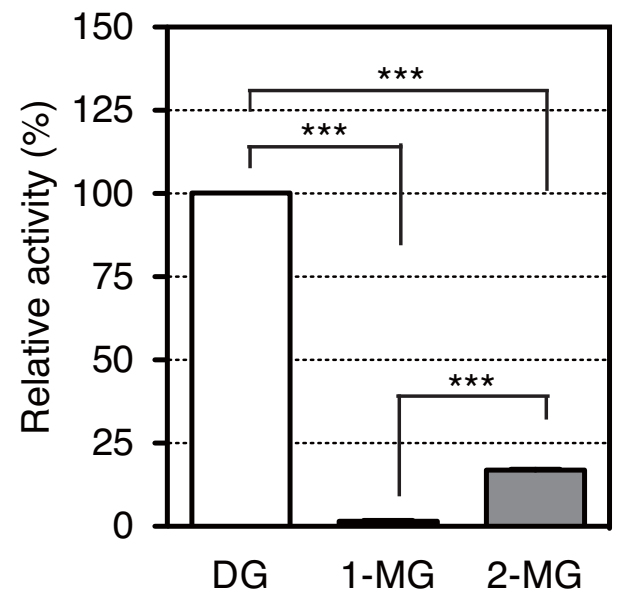

C

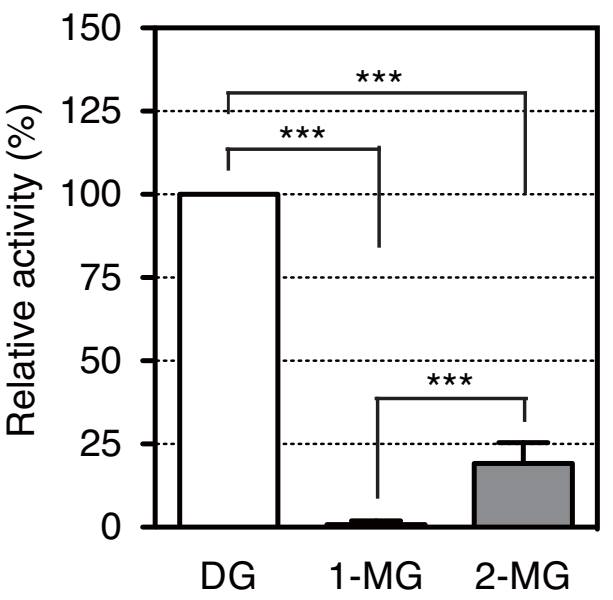


Fig. 2

A

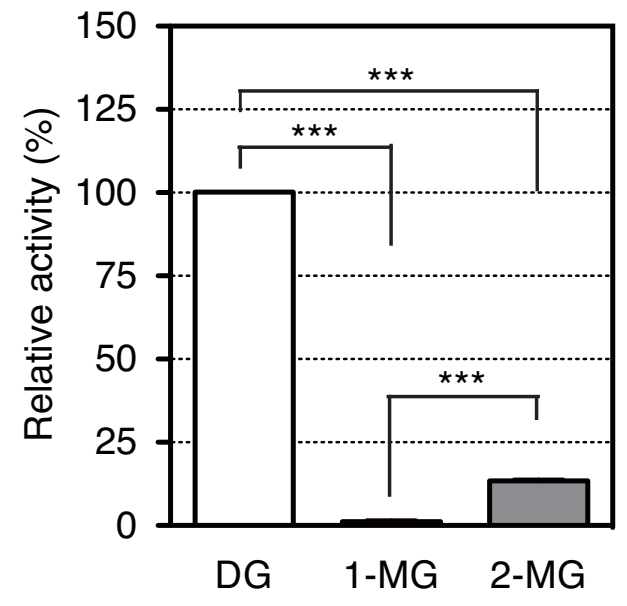

B

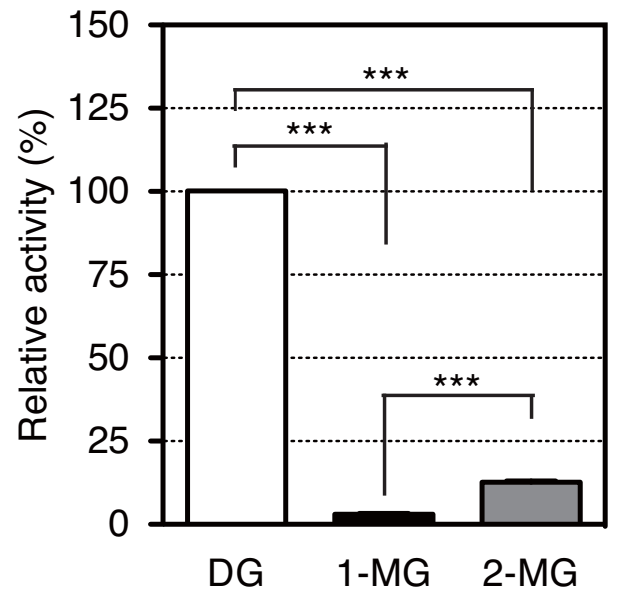

C

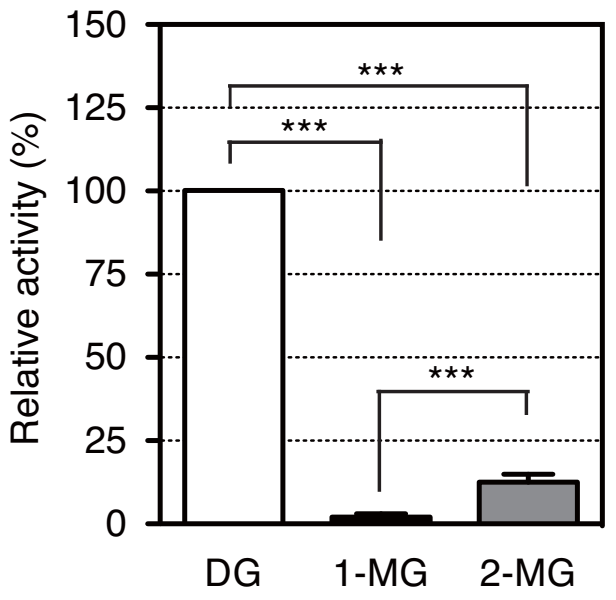



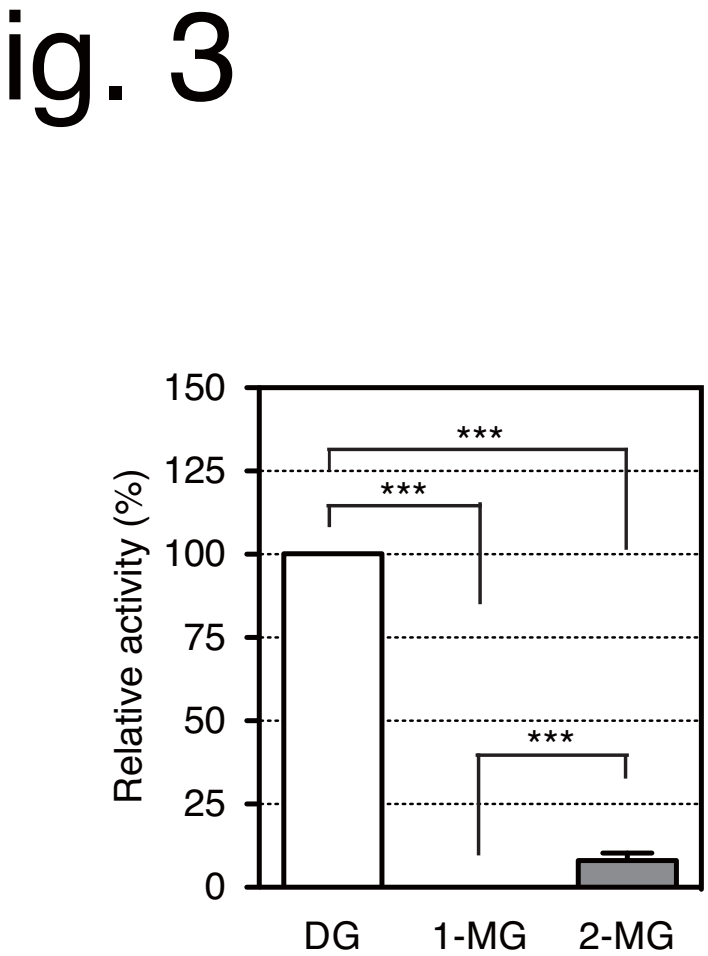
A

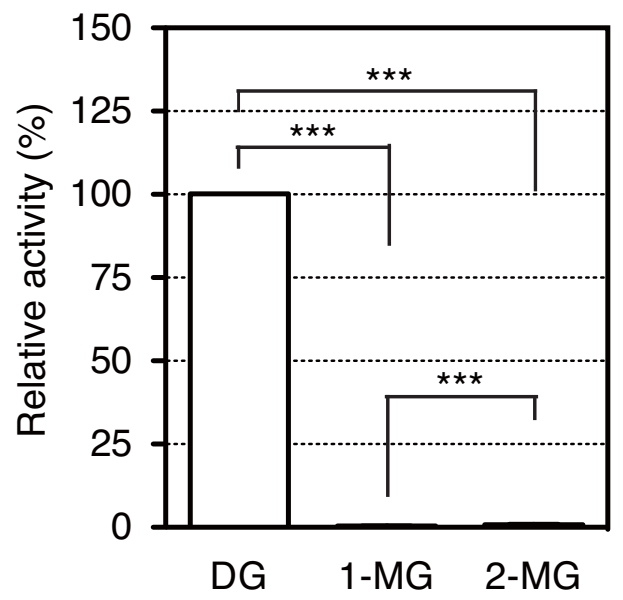

B

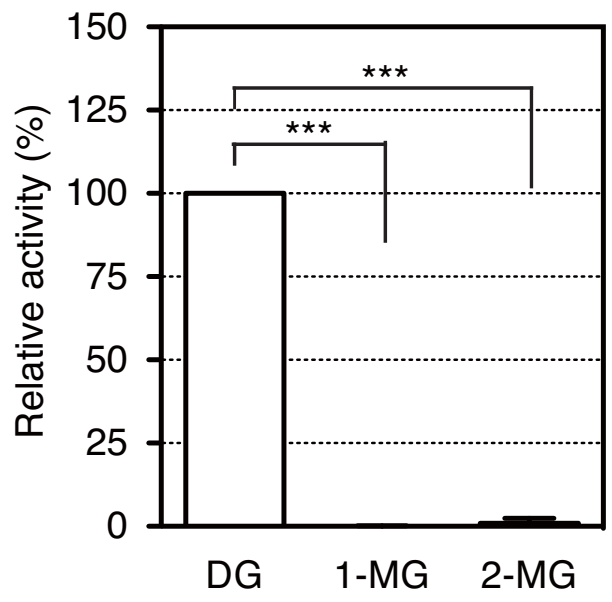



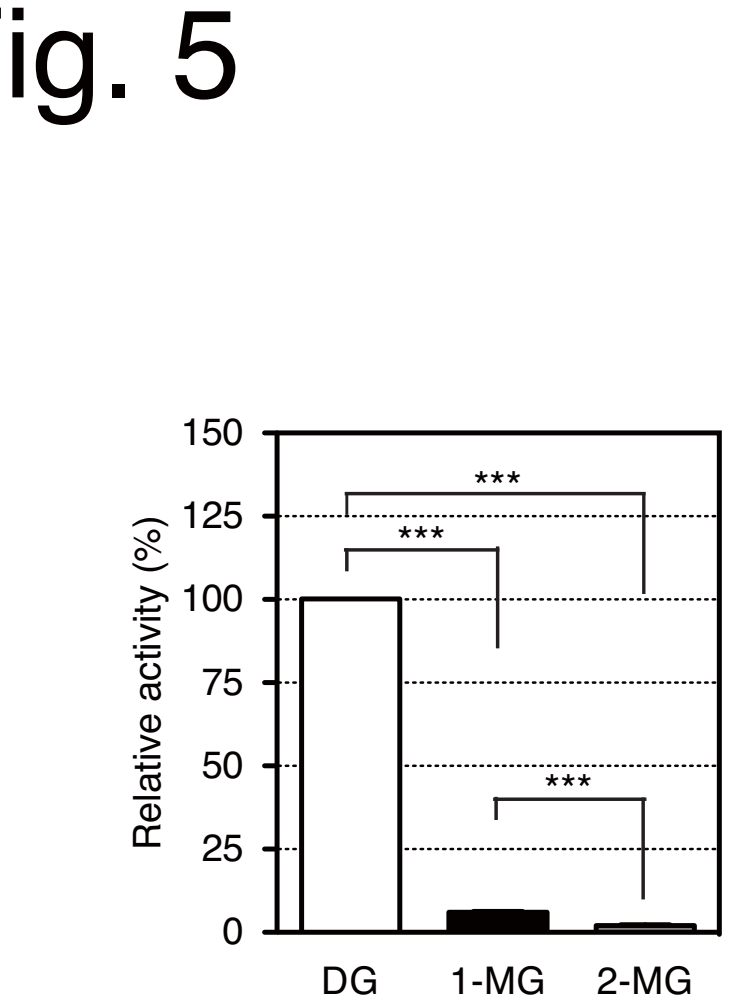
A

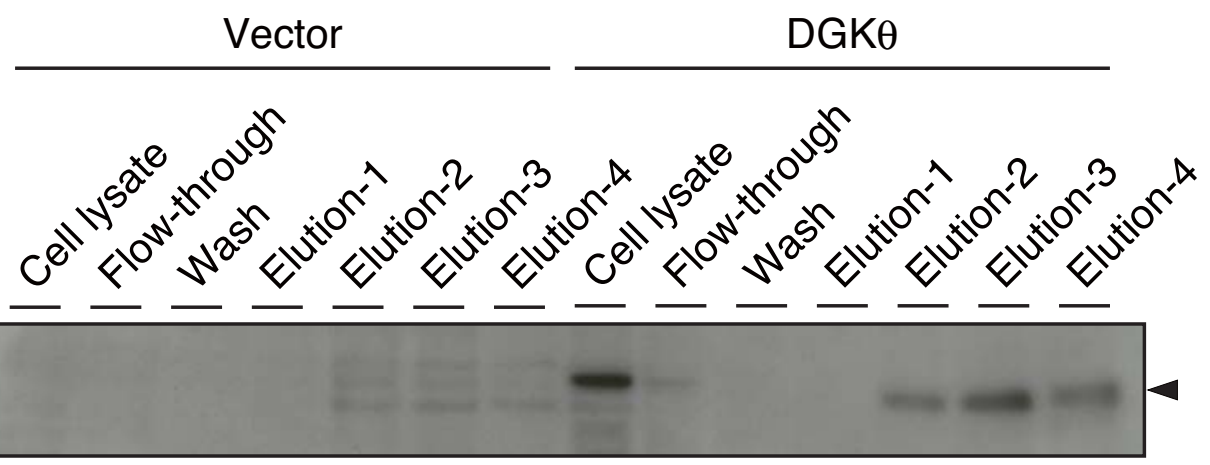

B

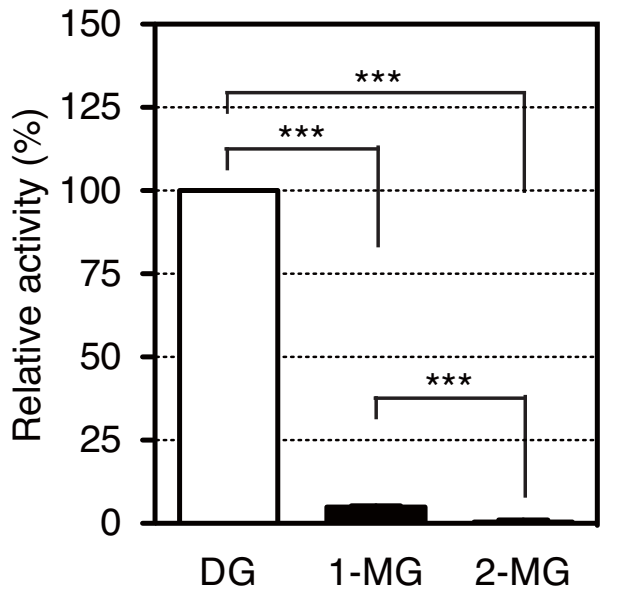




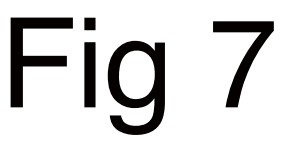

A
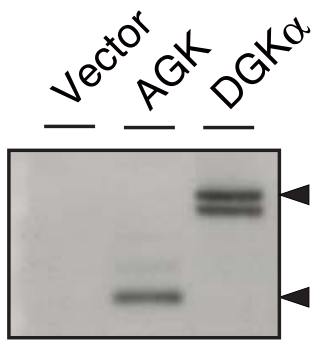
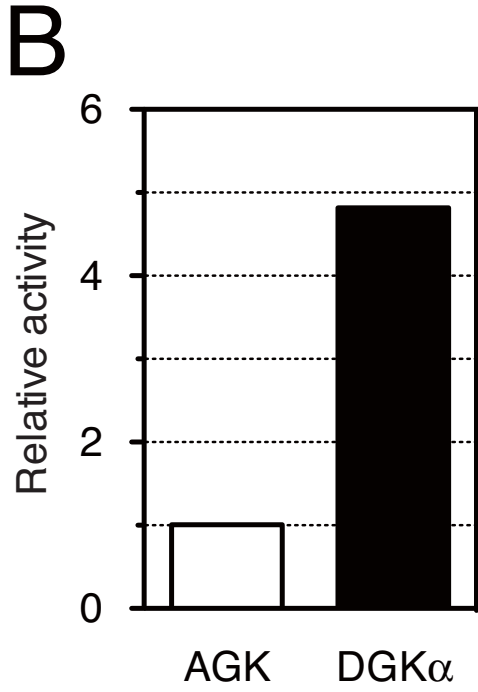
